\title{
Right Ventricular Perforation by a Defibrillator Lead Migrating to the Left Breast
}

\author{
Jerneja Tasic, ${ }^{1}$ Rok Zbacnik, ${ }^{2}$ Igor Zupan, ${ }^{1}$ Jus Ksela ${ }^{3}$ \\ Departments of ${ }^{1}$ Cardiology, ${ }^{2}$ Clinical Institute of Radiology, and ${ }^{3}$ Cardiovascular Surgery, University Medical Center Ljubljana, \\ Ljubljana, Slovenia
}

\section{ABSTRACT}

Cardiac perforation after an ICD implantation is a rare complication, with a reported incidence between 0.6-5.2\%. Its manifestation might be acute, subacute, or delayed, with an acute perforation occurring within the first 24 hours after implantation, frequently accompanied by severe clinical signs, while subacute and delayed perforations have a more benign progression. Here, we report a case of a 69-year old patient with an acute right ventricular perforation by a defibrillator lead migrating all the way through the pericardium and thoracic wall into the left breast, with an unusually mild and benign clinical course, delaying prompt diagnosis and postponing subsequent surgical treatment. Heart perforation with a defibrillator electrode is a rare but dangerous complication, which may lead to pacing failure, cardiac tamponade, cardiogenic shock, and even death. Even with a benign clinical course, one must think of cardiac wall perforation at any time after device implantation, and a contrast enhanced computer tomography (CTA) must be performed if perforation is suspected. At re-implantation, the lead should be located at a different anatomical position within the RV, preferably at the interventricular septal site, and manipulation of the injury site within the RV avoided.

\section{INTRODUCTION}

Cardiac perforation after an ICD implantation is a rare complication, with a reported incidence between $0.6-5.2 \%$ [Hsu 2013]. While acute perforations develop within the first 24 hours after implantation, subacute and delayed perforations might develop days or even week or months after the primary surgical procedure and present with a more benign clinical progress [Khan 2005; Sterlinski 2009]. Although acute perforations generally present with a severe clinical course, they occasionally can have a benign display, which makes the accurate diagnosis easily overlooked and delaying the necessary medical and/or surgical treatment [Kirkfeld 2011; Hsu 2013].

Here, we report a case of a 69-year old patient with an acute right ventricular perforation by a defibrillator lead migrating through the pericardium and thoracic wall into the left breast, with an unusual mild clinical course, delaying

Received November 14, 2017; accepted fanuary 24, 2018.

Correspondence: Fus Ksela, MD, PhD, Department of Cardiovascular Surgery, University Medical Center Ljubljana, Zaloska cesta 7, 1000 Ljubljana, Slovenia; +386-1-522-8251 (e-mail:jus.ksela@kclj.si). prompt diagnosis and postponing an otherwise successful subsequent surgical treatment.

\section{CASE REPORT}

A 69-year old Caucasian female with nonischemic dilated cardiomyopathy and with frequent ventricular extrasystole was admitted to our department for further treatment. An implantable cardioverter defibrillator (ICD) implantation for primary prevention was indicated and the Biotronik Iforia VR ICD with an active fixation electrode LinoxSmart Dx 65/15 Pro MRI was implanted in a standardised surgical manner. The early postoperative course was uneventful. However, 12 hours after the surgery, the patient started to complain about mild pain under her left breast. Physical examination did not reveal any significant abnormalities, and the chest X-ray was normal with an appropriate lead position. The patient was dismissed from the hospital 24 hours after the surgery. However, the pain progressively escalated in subsequent days, and she was re-admitted to the emergency department 48 hours after her discharge. The chest X-ray was repeated and a slight migration of the electrode tip was noticed.

The patient was redirected to the pacemaker clinic, where the telemetric interrogation revealed malfunction of the implanted electrode, with no sensing and pacing in the right ventricle (RV), yet with normal sensing and pacing in the atrium, and normal lead impedances. The chest X-ray was repeated, revealing further migration of the electrode and the perforation of the RV was suspected. A transthoracic echocardiogram was performed promptly, revealing no signs of pericardial effusion. However, the exact location of the electrode could not be determined. Next, a contrast enhanced computer tomography (CTA) scan of the heart was performed, showing suitable position of the electrode in the atrium with an obvious malposition of the electrode in the ventricle. The tip of the lead had pierced the apex of the RV, further penetrating the pericardial space and the entire thickness of the thoracic wall, stopping subcutaneously in the soft tissue of the left breast with no signs of pericardial effusion or thoracic wall- or breast-hematoma (Figure 1).

Surgical lead extraction with RV repair was indicated. At the time of surgery, a lower mini-sternotomy to the sixth left intercostal space was performed and the apex of the heart visualized. The electrode perforating the RV and thoracic wall was clearly noticed (Figure 2). Next, the electrode was removed by careful retrograde traction, and the RV perforation wound was closed using several interrupted double armed pledget supported 3/0 Prolene sutures. The postoperative 
course was uneventful and three weeks later a defibrillator was re-implanted with the lead positioned and fixated to the $\mathrm{RV}$ septum. At one year after re-implantation, the patient is clinically stable, managed on an outpatient basis, with a stable lead position and proper function, and without any signs of RV dysfunction.

\section{DISCUSSION}

Signs and symptoms of cardiac perforation and electrical parameters of the perforated electrode depend on the exact location of the lead tip, and can vary considerably [Khan 2005; Sterlinski 2009; Kirkfeld 2011; Seil 2011; Banaszewski 2012; Hsu 2013]. Although acute perforations, developing within 24 hours after the implantation, generally present with a severe clinical course, they occasionally can have a benign display, which makes the accurate diagnosis easily overlooked or delayed, as in our patient. Since she presented with only mild symptoms, the presumably acute perforation was unrecognized for several days when the electrode had migrated into the breast tissue. Noteworthy, while sensing and pacing failure is commonly present with perforated leads, the electrode impedance, depending mainly on the surrounding tissue composition, may stay within normal ranges, even in profoundly displaced electrodes, as in our case [Seil 2011]. Several factors are known to lead to myocardial injury and cardiac perforation. The most prominent of these include: corticosteroid use, active fixation leads, low body-mass index, advanced age, longer fluoroscopy times, and excessive length of the electrode in the ventricle [Kirkfeld 2011; Seil 2011]. In
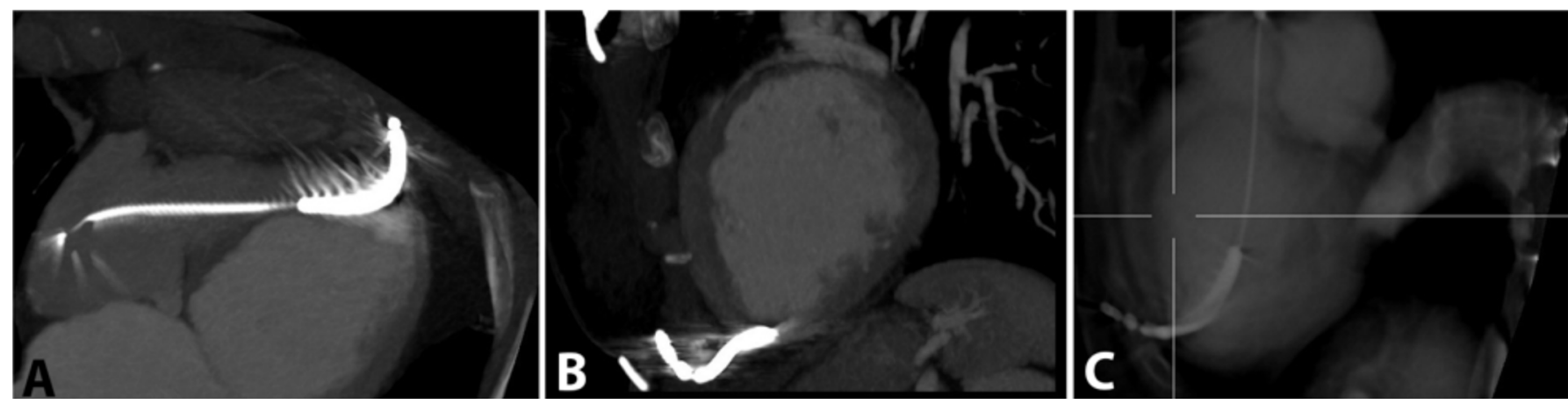

Figure 1: Preoperative findings (computed tomography of the heart): the ICD electrode perforating the myocardium, pericardium and the whole thickness of the thoracic wall, stopping subcutaneously in the soft tissue of the left breast with no signs of pericardial effusion or thoracic wall- or breast-hematoma (panel A: maximal intensity projection four-chamber view; panel B: maximal intensity projection modified long axis two chamber view; panel C: thick slab multi planar reconstruction modified long axis two-chamber view).

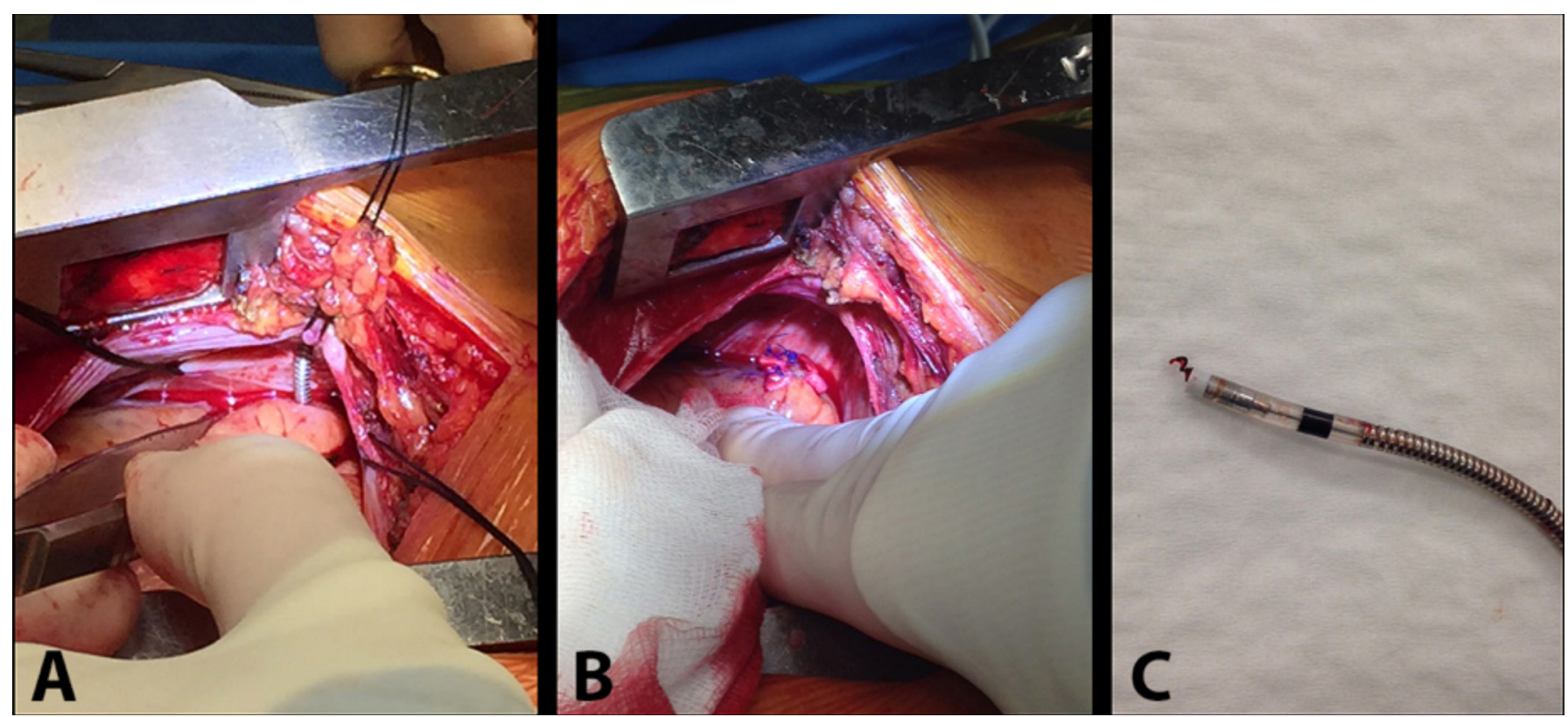

Figure 2: Intraoperative findings. Panel A: ICD electrode perforating the right ventricle and piercing the thoracic wall as seen during surgery. Panel B: surgical correction of the RV perforation wound. Panel C: tip of the extracted electrode. 
our patient, placement of an active fixation lead to the thinwalled RV apex was most probably the leading pathological mechanism, resulting in myocardial and thoracic wall perforation [Khan 2005; Seil 2011].

During the diagnostic workup, chest radiography and echocardiography are helpful in comparing the lead tip position and the lead curvature to that in the initial $x$-ray, and in evaluating the pericardial space for potential effusion, respectively, yet both diagnostic tools usually fail to observe lead tip migrations [Seil 2011]. CTA of the heart, precisely depicting the lead tip position and accurately delineating intrathoracic patho-anatomical status, is therefore the diagnostic modality of choice.

In asymptomatic patients with normal pacemaker function and no adjacent organ damage, the necessity of lead extraction is controversial [Seil 2011]. In symptomatic individuals without pericardial effusion a simple traction under echocardiographic and fluoroscopic guidance with surgical backup can be considered. In patients with cardiac tamponade or extremely migrated leads, a conventional surgical procedure with direct heart visualization is recommended [Seil 2011; Hsu 2013]. Postoperatively, close echo-cardiographic observation should be performed to exclude delayed tamponade [Kirkfeld 2011; Seil 2011]. At the time of ICD re-implantation, the lead should be located at a different anatomical position within the RV, preferably at the interventricular septal site [Seil 2011].

\section{CONCLUSION}

To conclude, heart perforation with a defibrillator electrode is a rare but dangerous complication, which, if undiagnosed, may lead to pacing failure, cardiac tamponade, cardiogenic shock, and even death. Even with a benign clinical course, one must think of cardiac wall perforation at any time after device implantation, and a CTA must be performed if perforation is suspected. At re-implantation, the lead should be located at a different anatomical position within the RV, preferably at the interventricular septal site, and manipulation of the injury site within the RV avoided.

\section{REFERENCES}

Banaszewski M, Stępińska J. 2012. Right heart perforation by pacemaker leads. Arch Med Sci 8:11-13.

Hsu JS, Varosy PD, Bao H, et al. 2013. Cardiac perforation from implantable cardioverter-defibrillatorlead placement. Insights from the National cardiovascular data registry. Circ Cardiovasc Qual Outcomes 6:582-590.

Khan MN, Joseph G, Khaykin Y, Ziada KM, Wilkoff BL. 2005. Delayed lead perforation: a disturbing trend. Pacing Clin Electrophysiol 28:251-3.

Kirkfeldt RE, Johansen JB, Nohr EA, et al. 2011. Risk factors for lead complications in cardiac pacing: A population-based cohort study of 28,860 Danish patients. Heart Rhythm 8:1622-1628.

Seil O. Cardiac Perforation Associated with a Pacemaker or ICD Lead, Modern Pacemakers - Present and Future, Prof. Mithilesh R Das (Ed.), 2011. ISBN: 978-953-307-214-2.

Sterlinski M, Przybylski A, Maciag, et al. 2009. Subacute cardiac perforations associated with active fixation leads. Europace 11: 206-212. 